\title{
Comparison of follitropin $\beta$ administered by a pen device with follitropin $\beta$ administered by a conventional syringe in patients undergoing IVF-ET
}

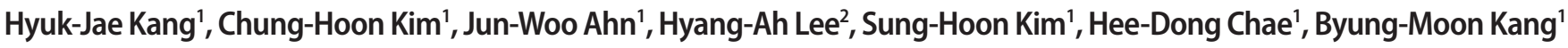

'Division of Reproductive Endocrinology and Infertility, Department of Obstetrics and Gynecology, Asan Medical Center, University of Ulsan College of Medicine, Seoul; ${ }^{2}$ Department of Obstetrics and Gynecology, Kangwon National University Hospital, Kangwon National University School of Medicine, Chuncheon, Korea

Objective: To compare the effectiveness and convenience of a pen device for the self-administration of follitropin $\beta$ with a conventional syringe delivering follitropin $\beta$ solution in patients undergoing IVF-ET.

Methods: GnRH agonist long protocol was used for controlled ovarian stimulation (COS) in all subjects. A total of 100 patients were randomized into the pen device group or the conventional syringe group on the first day of COS. Local tolerance reactions were assessed within 5 minutes, at 1 hour and at 3 hours after each injection. On the day of hCG injection, patients were asked to rate their overall pain and convenience experienced with self-injection on a visual anlaogue scale (VAS).

Results: There were no differences in patients' characteristics between the two groups. The duration of COS was significantly shorter in the pen device group than in the conventional syringe group. Patients included in the pen device group needed a significantly smaller amount of follitropin $\beta$. However, no differences between the two groups were found in IVF results and pregnancy outcome. The incidence of local pain within 5 minutes, at 1 hour and at 3 hours after the injection was significantly lower in the pen device group. VAS scores indicated that injections using the pen device were significantly less painful and more convenient.

Conclusion: The pen device for self-administration of follitropin $\beta$ is less painful, safer and more convenient for the patients, and can be more effective because of the shorter duration and smaller dose of follitropin $\beta$ when compared with the conventional syringe.

Keywords: Ovulation Induction; In Vitro Fertilization; Injections, Subcutaneous; Follicle Stimulating Hormone, Beta Subunit; Human

\section{Introduction}

Urinary FSH had been replaced by recombinant human FSH (rhFSH)

Received: Sep 5, 2010 · Revised: Oct 27, 2010 · Accepted: Nov 11, 2010 Corresponding author: Chung-Hoon Kim

Division of Reproductive Endocrinology and Infertility, Department of Obstetrics and Gynecology, Asan Medical Center, University of Ulsan College of Medicine, 388-1 Pungnap-dong, Songpa-gu, Seoul 138-736, Korea

Tel: +82-2-3010-3639 Fax:+82-2-476-7331 E-mail:chnkim@amc.seoul.kr

* Presented at 61th Annual Meeting of American Society of Reproductive Medicine in Montreal, Canada.

*This work was supported by the Organon grant 2003-0219.

This is an Open Access article distributed under the terms of the Creative Commons Attribution Non-Commercial License (http://creativecommons.org/licenses/by-nc/3.0/) which permits unrestricted non-commercial use, distribution, and reproduction in any medium, provided the original work is properly cited. because rhFSH has a lower risk of immunogenicity and infection than that of the urinary products. Recombinant products are highly pure ( $>99 \%$ ), and indeed, no antibodies to FSH have been detected in serum samples from patients who have injected rhFSH. Therefore, the use of this recombinant product has resulted in more efficient and predictable ovulation induction or controlled ovarian stimulation (COS). Additionally, rhFSH has been shown to be suitable for subcutaneous (SC) administration and SC injections allow for easy self-administration by patients $[1,2]$.

RhFSH was first available as a freeze-dried lyophilized powder, which had to be dissolved in water for injection before administration. Recently, rhFSH has been made available as a premixed ready-to-use solution and this product has been considered more "patient friend- 
ly". A subsequent advance in patient-friendly regimens is the development of rhFSH solution, which is stored in cartridge form for administration with a pen device. This multiple-use pen device for SC self-administration of rhFSH, which is approved under the brand name Puregon Pen has been available in Europe since 2002. This pen injector is an adapted insulin pen, which has been shown to be better accepted by diabetes patients in comparison with conventional syringes, as it provides for an easier, safer, more accurate and more discrete insulin injection [3].

This study was performed to compare the effectiveness and convenience of a pen device for the self-administration of rhFSH (follitropin ß) with a conventional syringe delivering $\mathrm{rhFSH}$ solution in patients undergoing COS for IVF-ET.

\section{Methods}

This study was a prospective, randomized, single center, group comparative study. A total of 100 infertile Korean women who were scheduled for IVF-ET with or without ICSI were enrolled in this study and they were divided into the pen device group or the conventional syringe group by a computer-generated randomization list using random numbers on the first day of COS. The patients were 20-39 years of age at the time of screening, and had normal ovulatory cycles (24-35 days in length), had a body mass index (BMI) between 18 and $29 \mathrm{~kg} / \mathrm{m}^{2}$, and were willing to self-inject rhFSH.

Patients who had a history of current endocrine abnormalities, such as polycystic ovary syndrome, hyperprolactinemia, and a previous history of IVF-ET in which less than three oocytes were retrieved were excluded in this study. Patients who had any abnormalities that would interfere with adequate stimulation, previous hospitalization due to severe ovarian hyperstimulation syndrome (OHSS), history of previous (within 12 months) or current abuse of alcohol or drugs were also excluded from this study.

All patients received the same $\mathrm{COS}$ regimen, $\mathrm{GnRH}$ agonist luteal long protocol using triptorelin acetate (Decapeptyl; Ferring, Malmo, Sweden). After an absence of ovarian activity was confirmed by transvaginal ultrasound and/or serum estradiol levels after the administration of triptorelin acetate for at least 2 weeks, ovarian stimulation with rhFSH was carried out in both groups, starting with 200 or 250 IU follitropin $\beta$.

On the first day of ovarian stimulation, all subjects were randomized to self-administer rhFSH using a pen device (Puregon Pen; Organon, Oss, Netherland) equipped with a needle sized $29 \mathrm{G} \times 13 \mathrm{~mm}$ or a conventional syringe equipped with a needle sized $25 \mathrm{G} \times 16 \mathrm{~mm}$ by a computer generated randomization list using random numbers. On the first day of ovarian stimulation, patients were instructed how to self-inject subcutaneously into the abdominal wall around the umbilicus with a pen device or a conventional syringe and supervised in order to ensure correct self-injection.

After stimulation day 5 , the dose of follitropin $\beta$ was adjusted according to the individual ovarian response monitored by transvaginal ultrasound examinations. When one leading follicle reached 18 $\mathrm{mm}$ in diameter or 2 or more follicles reached $17 \mathrm{~mm}$, oocyte maturation was triggered with 10,000 IU of hCG (Pregnyl; Organon) administered as a single intramuscular injection. Transvaginal ultrasound-guided oocyte retrieval was performed 36 hours after hCG administration. Embryos were transferred 3 days after oocyte retrieval. Luteal support was provided by administering $90 \mathrm{mg}$ of vaginal progesterone gel (Crinone gel 8\%; Serono SA, Geneva, Switzerland) once daily from the day of oocyte retrieval. Serum $\beta$-hCG levels were measured 11 or 12 days after embryo transfer. Pregnancies were confirmed by rising serum $\beta$-hCG concentrations and transvaginal ultrasonographic evidence of a gestational sac. If pregnancy was diagnosed, vaginal progesterone administration was continued for another 5 to 7 weeks. Clinical pregnancy was defined as the presence of a gestational sac by ultrasonography, while the miscarriage rate per clinical pregnancy was defined as the proportion of patients who failed to continue development before reaching 20 weeks of gestation among all clinical pregnancies.

\section{Local tolerance and convenience assessment}

After every injection of follitropin $\beta$, the severity of local tolerance symptoms was assessed by the patients themselves and recorded in a'local tolerance diary book', within 5 minutes, at 1 hour and at 3 hours after injection. Local tolerance items scored were pain, redness, itching, bruising and swelling. The severity of symptoms was scored as none, mild, moderate, or severe. The incidence of each local tolerance item was compared between the pen device group and the conventional syringe group. Incidence indicates the percentage of subjects with mild, moderate or severe local tolerance symptoms for each item at each assessment. Additionally, on the day of hCG administration, patients were asked to rate their overall pain and convenience experienced with this self-injection method on a visual anlaogue scale (VAS). The VAS ranged from 0 (severe pain, not convenient) to 10 (no pain, very convenient).

\section{Statistical analysis}

The mean value was expressed as the mean \pm standard deviation (SD). A Student's $t$-test was used to compare the mean values. The chi-square test and Fisher's exact test were used for the comparison of fractions. Statistical significance was defined as $p<0.05$. All analyses were performed using SPSS ver. 11.0 (SPSS Inc., Chicago, IL, USA). 
Table 1. Patients' characteristics

\begin{tabular}{lccc}
\hline & Pen & Syringe & $p$-value \\
\hline No. of patients & 50 & 50 & \\
Patients'age (yr) & $33.2 \pm 3.5$ & $33.0 \pm 3.5$ & NS \\
Husbands'age (yr) & $37.8 \pm 6.2$ & $38.2 \pm 5.9$ & NS \\
Infertility duration (yr) & $3.4 \pm 2.3$ & $3.2 \pm 1.9$ & NS \\
Patients with primary infertility & $16(32.0)$ & $18(36.0)$ & NS \\
BMI (kg/m ${ }^{2}$ ) & $25.6 \pm 2.5$ & $25.5 \pm 2.4$ & NS \\
Endocrine profile & & & \\
Basal FSH (mlU/mL) & $6.2 \pm 1.4$ & $6.0 \pm 1.3$ & NS \\
Basal LH (mlU/mL) & $5.4 \pm 1.5$ & $5.3 \pm 1.6$ & NS \\
T (ng/mL) & $0.3 \pm 0.1$ & $0.3 \pm 0.1$ & NS \\
FreeT (pg/mL) & $0.5 \pm 0.2$ & $0.4 \pm 0.2$ & NS \\
SHBG (nmol/L) & $52.4 \pm 12.4$ & $50.8 \pm 13.3$ & NS \\
Estradiol (pg/mL) & $29.8 \pm 8.4$ & $29.2 \pm 9.6$ & NS \\
Prolactin (ng/mL) & $13.4 \pm 3.8$ & $14.3 \pm 3.5$ & NS \\
Indications & & & \\
Tubal/peritoneal factor & $22(44.0)$ & $21(42.0)$ & NS \\
Male factor & $16(32.0)$ & $18(36.0)$ & NS \\
Unexplained & $12(24.0)$ & $11(22.0)$ & NS \\
\hline
\end{tabular}

Values are presented as mean \pm SD or number (\%).

$\mathrm{NS}$, not significant; BMI, body mass index; $\mathrm{FSH}$, follicle stimulating hormone; LH, luteinizing; T, testosterone; SHBG, sex hormone binding globulin.

\section{Results}

There were no differences in the age of patients and spouses, BMl, infertility duration, the rate of the patients with primary infertility, basal endocrine profile and indications for IVF/ICSI between the two groups (Table 1). The duration of COS with rhFSH was significantly shorter in the pen device group than in the conventional syringe group $(p<0.05)$. Patients included in the pen device group needed significantly less rhFSH, with $2,091.0 \pm 318.8$ IU compared with $2,248.0 \pm 405.0 \mathrm{IU}$ in the conventional syringe group $(p<0.05)$. However, no differences were found between the two groups in the numbers of oocytes retrieved, mature oocytes, fertilized oocytes, grade I/ Il embryos, frozen embryos and transferred embryos. Also, there were no differences between the two groups in the clinical pregnancy rate, implantation rate, miscarriage rate, multiple pregnancy rate and the incidence of severe OHSS (Table 2).

The incidence of local pain within 5 minutes after injection was significantly lower in the pen device group $(p<0.05)$. The incidence of local redness within 5 minutes after injection was also significantly lower in the pen device group $(p<0.05)$. The incidence of local pain at 1 hour and 3 hours after injection were significantly lower in the pen device group $(p<0.05, p<0.05)$ (Figure 1). The mean overall pain score assessed by VAS was significantly higher in the pen device group than in the conventional syringe group ( $9.3 \pm 0.9 \mathrm{vs.} 8.6 \pm 1.4 ; p<0.01)$,
Table 2. Comparison of controlled ovarian stimulation results and IVF outcome

\begin{tabular}{lccc}
\hline & Pen & Syringe & $p$-value \\
\hline No. of cycles & 50 & 50 & \\
No. of ICSI cycle & $15(30.0)$ & $16(32.0)$ & NS \\
Duration of COS (day) & $8.7 \pm 1.1$ & $9.3 \pm 1.3$ & $p<0.05$ \\
Total dose of rhFSH (IU) & $2,091.0 \pm 318.8$ & $2,248.0 \pm 405.0$ & $p<0.05$ \\
No. of oocytes retrieved & $9.8 \pm 3.0$ & $9.6 \pm 2.7$ & NS \\
No. of Mll oocytes & $8.9 \pm 3.0$ & $8.7 \pm 2.8$ & NS \\
No. of fertilized oocytes & $8.8 \pm 2.0$ & $8.6 \pm 2.8$ & NS \\
No. of grade I, Il oocytes & $3.2 \pm 1.5$ & $2.9 \pm 1.3$ & NS \\
No. of frozen embryos & $1.7 \pm 1.3$ & $1.5 \pm 1.1$ & NS \\
No. of embryo transferred & $2.9 \pm 0.3$ & $3.0 \pm 0.3$ & NS \\
Clinical pregnancy rate & $17 / 50(34.0)$ & $18 / 50(36.0)$ & NS \\
Implantation rate & $19 / 145(13.1)$ & $20 / 150(13.3)$ & NS \\
Miscarriage rate & $4 / 17(23.5)$ & $4 / 18(22.2)$ & NS \\
Multiple pregnancy rate & $2 / 17(11.8)$ & $2 / 18(11.1)$ & NS \\
Incidence of severe OHSS & $1 / 50(2)$ & $2 / 50(4)$ & NS \\
\hline
\end{tabular}

Values are presented as number (\%) or mean \pm SD.

ICSI, intracytoplasmic sperm injection; NS, not significant; COS, controlled ovarian stimulation; rhFSH, recombinant human follicle stimulating hormone; MII, metaphase II; OHSS, ovarian hyperstimulation syndrome.

and the convenience score was also significantly higher in the pen device group ( $9.4 \pm 0.7$ vs. $8.0 \pm 1.6 ; p<0.001$ ).

\section{Discussion}

The introduction of a pen device for the administration of recombinant insulin in the treatment of diabetes resulted in significant improvements for patients in terms of local discomfort, accurate dosing and convenience to the patients [4-6]. The development of the pen device made it possible for patients to self-inject medication, reduce the number of visits to the clinic and administer accurate doses of drugs. A similar pen device for the injection of gonadotropin was deemed necessary for the convenience and effectiveness of the patients. A study by Voortman et al. [7] demonstrated that injections of similar amounts of follitropin $\beta$ using either a dissolved cake with a conventional syringe or a ready-for-use solution with a pen device were bio-equivalent, after correction for injection losses by weighing the amount injected with a normal syringe. A difference of approximately $18 \%$ was found between serum FSH concentrations obtained using the two formulations, which was caused by differences between the anticipated volume and the actual volume injected with the normal syringe [7]. Another study showed that follitropin $\beta$ administered with a pen device is not only well tolerated but also more efficacious with respect to ovarian stimulation outcome when compared with the conventional syringe [8]. In our study, the number of administra- 


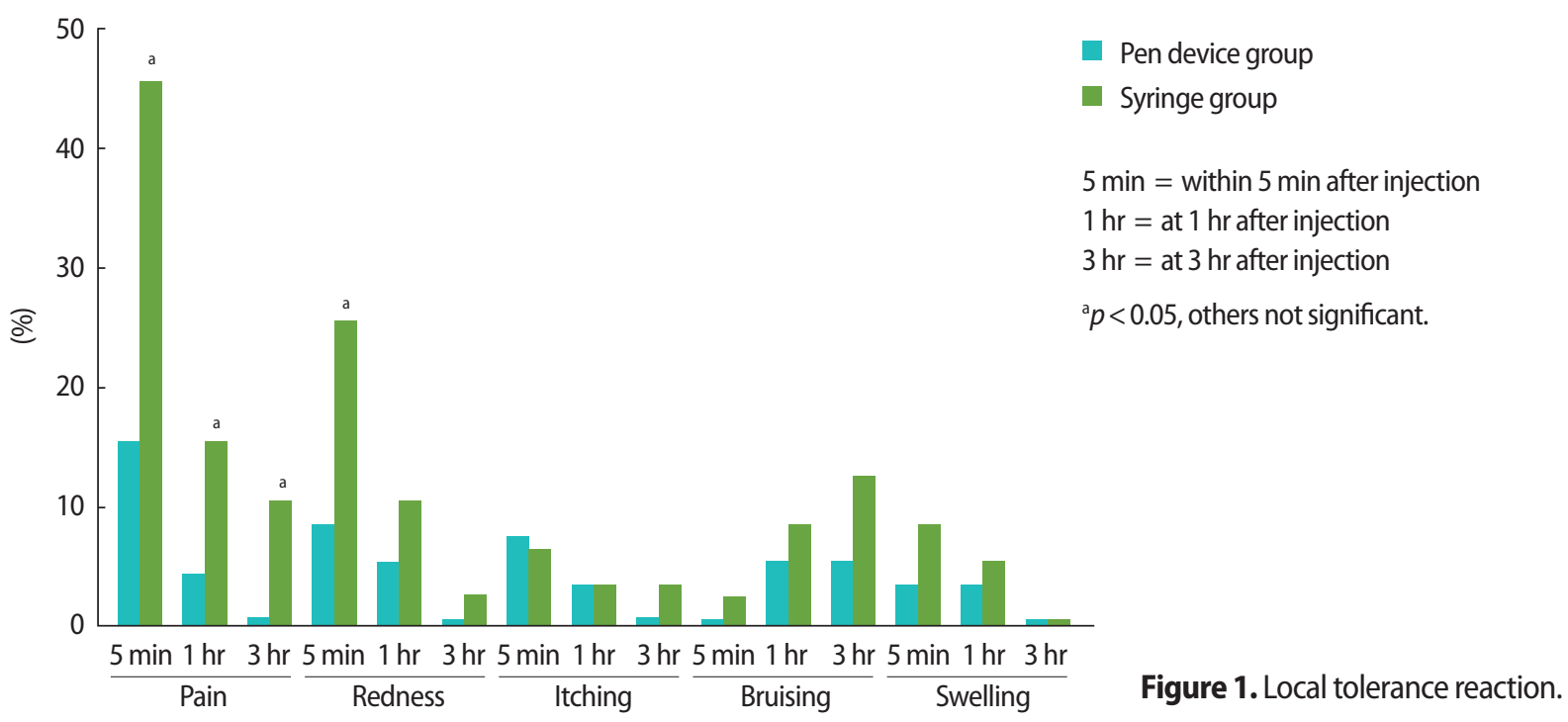

tion days and amount of follitropin $\beta$ required for $\operatorname{COS}$ were significantly reduced in the pen device group over those in the conventional syringe group. These results suggest that the difference between the anticipated volume and the actual volume injected was higher in the conventional syringe group and that an administration of rhFSH by a pen device reduced injection losses.

A previous study showed that injection pain is experienced less frequently when follitropin $\beta$ is administered by a pen device than when follitropin $\alpha$ is administered with a conventional syringe [9]. In this study, the reason the local pain at the injection site was lower in the pen device group may have been that the pen device was equipped with a finer and shorter needle and injected a smaller volume of rhFSH [9]. In our study, patients also experienced local pain less frequently after injection when they used the pen device for injections. Overall pain assessed by VAS was also significantly lower in the pen device group than in the syringe group.

None of the patients withdrew from our study because of the pain or other side effects. In addition, the incidence of severe OHSS was comparable between the two groups. Therefore, the pen device delivering rhFSH is judged to be at least as safe as the conventional syringe. Moreover, the present study demonstrated that the pen device is more convenient and easy to handle, when compared with the conventional syringe. The pen device delivering follitropin $\beta$ permits accurate titration of rhFSH doses in increments of $25 \mathrm{IU}$. Individualized treatment for $\operatorname{COS}$ was therefore possible at the minimal effective dose.

In conclusion, the pen device for self-administration of follitropin $\beta$ is safe, less painful and more convenient for patients undergoing COS, and can be more effective because of the shorter duration and smaller dose of follitropin $\beta$, when compared with a conventional syringe. A reduction of painful injections and the treatment period, and the convenient administration of rhFSH can make IVF treatment easier and more patient-friendly.

\section{Conflict of interest}

No potential conflict of interest relevant to this article was reported.

\section{References}

1. Howles CM, Loumaye E, Giroud D, Luyet G. Multiple follicular development and ovarian steroidogenesis following subcutaneous administration of a highly purified urinary FSH preparation in pituitary desensitized women undergoing IVF: a multicentre European phase III study. Hum Reprod 1994;9:424-30.

2. Wikland M, Borg J, Hamberger L, Svalander P. Simplification of IVF: minimal monitoring and the use of subcutaneous highly purified FSH administration for ovulation induction. Hum Reprod 1994;9:1430-6.

3. Kadiri A, Chraibi A, Marouan F, Ababou MR, el Guermai N, Wadjinny $A$, et al. Comparison of NovoPen 3 and syringes/vials in the acceptance of insulin therapy in NIDDM patients with secondary failure to oral hypoglycaemic agents. Diabetes Res Clin Pract 1998:41:15-23.

4. Bohannon NJ. Insulin delivery using pen devices. Simple-to-use tools may help young and old alike. Postgrad Med 1999;106:57-8.

5. Robertson KE, Glazer NB, Campbell RK. The latest developments in insulin injection devices. Diabetes Educ 2000;26:135-8.

6. Bohannon NJ, Ohannesian JP, Burdan AL, Holcombe JH, Zagar A. Patient and physician satisfaction with the Humulin/Humalog Pen, a new 3.0-mL prefilled pen device for insulin delivery. Clin Ther 2000;22:1049-67. 
7. Voortman G, van de Post J, Schoemaker RC, van Gerven JM. Bioequivalence of subcutaneous injections of recombinant human follicle stimulating hormone (Puregon(R)) by Pen-injector and syringe. Hum Reprod 1999;14:1698-702.

8. Rama Raju GA, Suryanarayana K, Jaya Prakash G, Murali Krishna K. Comparison of follitropin-beta administered by a pen device with conventional syringe in an ART programme: a retrospective study. J Clin Pharm Ther 2008;33:401-7.

9. Craenmehr E, Bontje PM, Hoomans E, Voortman G, Mannaerts BM. Follitropin-beta administered by pen device has superior local tolerance compared with follitropin-alpha administered by conventional syringe. Reprod Biomed Online 2001;3:185-9. 